\title{
THE SHARP BOUND FOR THE DEFORMATION OF A DISC UNDER A HYPERBOLICALLY CONVEX MAP
}

\author{
ROGER W. BARNARD, LEAH COLE, KENT PEARCE, AND G. BROCK \\ WILLIAMS
}

\begin{abstract}
We complete the determination of how far convex maps can deform discs in each of the three classical geometries. The euclidean case was settled by Nehari in 1976, and the spherical case by Mejía and Pommerenke in 2000. We find the sharp bound on the Schwarzian derivative of a hyperbolically convex function and thus complete the hyperbolic case. This problem was first posed by Ma and Minda in a series of papers in the 1980's. Mejía and Pommerenke then produced partial results and a conjecture as to the extremal function in 2000. Their function maps onto a domain bounded by two proper geodesic sides, a "hyperbolic strip." Applying a generalization of the Julia variation and a critical Step Down Lemma, we show that there is an extremal function mapping onto a domain with at most two geodesic sides. We then verify using special function theory that among the remaining candidates, Mejía and Pommerenke's two-sided domain is in fact extremal. This correlates nicely with the euclidean and spherically convex cases in which the extremal is known to be a map onto a two-sided "strip."
\end{abstract}

\section{Introduction}

Hyperbolic convexity is a natural generalization of euclidean convexity; a region $G$ in the Poincaré model $\mathbb{D}$ of the hyperbolic plane is hyperbolically convex if for any two points in $G$, the hyperbolic geodesic segment between them lies entirely in $G$. Such regions arise naturally in Teichmüller theory, for example, since the fundamental domains of Fuchsian groups are hyperbolically convex $[5,7]$.

A conformal map $f: \mathbb{D} \rightarrow \mathbb{D}$ is hyperbolically convex if its range is hyperbolically convex. Hyperbolically convex functions have been extensively studied by Ma and Minda [8,9] and Mejía and Pommerenke [10-14], as well as Beardon [5] and Solynin $[21,22]$, among others. One frequently cited open problem is to find for hyperbolically convex functions a sharp bound on the Schwarz norm

$$
\left\|S_{f}\right\|_{\mathbb{D}}=\sup \left\{\left|S_{f}(z)\right| \eta_{\mathbb{D}}^{-2}(z): z \in \mathbb{D}\right\},
$$

where $\eta_{\mathbb{D}}(z)=\frac{1}{1-|z|^{2}}$ is the hyperbolic density of $\mathbb{D}$ and $S_{f}$ is the Schwarzian derivative

$$
S_{f}=\left(\frac{f^{\prime \prime}}{f^{\prime}}\right)^{\prime}-\frac{1}{2}\left(\frac{f^{\prime \prime}}{f^{\prime}}\right)^{2} .
$$

The Schwarz norm of an analytic function $f$ has long been a primary tool in understanding its geometric behavior. For example, $\left\|S_{f}\right\|_{\mathbb{D}}=0$ if and only if $f$ is a Möbius transformation. Thus $\left\|S_{f}\right\|_{\mathbb{D}}$ is thought of as measuring how closely the geometric behavior of $f$ resembles that of a Möbius transformation. Since the image of $\mathbb{D}$ under a Möbius transformation must be a disc, $\left\|S_{f}\right\|_{\mathbb{D}}$ also measures 

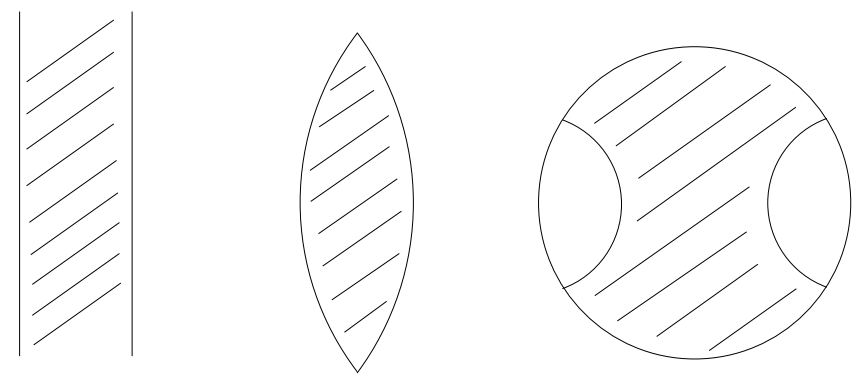

FIGURE 1. The extremal domains for maximizing the Schwarz norm in euclidean (left), spherical (center), and hyperbolic (right) geometry.

the difference between the conformal geometry of $f(\mathbb{D})$ and that of a disc. Lehto has used this idea to great effect, producing a pseudo-metric on the set of all simply connected proper subdomains of $\mathbb{C}$. See Lehto's book [7], for example, for a detailed discussion.

Nehari showed that if $f(\mathbb{D})$ is convex (in the euclidean sense), then $\left\|S_{f}\right\|_{\mathbb{D}} \leq 2$, with equality if and only if $f(\mathbb{D})$ is an "infinite strip" bounded by two parallel lines [18]. Similarly, Mejía and Pommerenke showed that the extremal domain for spherically convex functions is a "spherical strip" [11].

The problem of finding a similar bound for the Schwarz norm of hyperbolically convex functions has been intensely studied by a number of authors, including Ma, Minda, Mejía, Pommerenke and Vasilev [8-10,13-15]. Mejía and Pommerenke [10] found partial results on the bound and conjectured that the extremal value of $\left\|S_{f}\right\|_{\mathbb{D}}$ is attained by a map of the form

$$
f_{\alpha}(z)=\tan \left(\alpha \int_{0}^{z}\left(1-2 \xi^{2} \cos 2 \theta+\xi^{4}\right)^{-1 / 2} d \xi\right)
$$

where $\alpha=\frac{\pi}{2 K(\cos \theta)}$, and $K$ is the elliptic integral of the first kind. The range of $f_{\alpha}$ is a "hyperbolic strip" bounded by two geodesics through $\pm i \tanh \left(\frac{\pi K(\sin \theta)}{4 K(\cos \theta)}\right)$ and perpendicular to the imaginary axis. See Figure 1.

In this paper, we prove the following theorem, thus verifying Mejía and Pommerenke's conjecture and completing the classification of the extremal domains for the Schwarzian in all three of the classical geometries.

THEOREM 1.1. The maximal value of the Schwarz norm for hyperbolically convex functions is $S_{f_{\alpha}}(0)$, where

$$
f_{\alpha}(z)=\tan \left(\alpha \int_{0}^{z}\left(1-2 \xi^{2} \cos 2 \theta+\xi^{4}\right)^{-1 / 2} d \xi\right), \quad \alpha=\frac{\pi}{2 K(\cos \theta)},
$$

$K$ is the elliptic integral of the first kind, and $\alpha$ is chosen so that $\cos \theta$ is the unique critical point of the function

$$
g(s)=4 s^{2}-2+\frac{\pi^{2}}{2 K^{2}(s)}
$$

on $(0,1)$.

A computer calculation produces a maximal value for the Schwarz norm for a hyperbolically convex function of approximately 2.383635 . 
REMARK 1.1. Our variational techniques are used to argue that if an extremal function exists, it must have certain properties. To guarantee the existence of an extremal function, we restrict our attention to a dense collection of compact classes (hyperbolically convex functions which map onto domains with at most a fixed number of (proper) sides) and argue that an extremal function exists for these classes. Our methods show that the map $f_{\alpha}$ defined in (1.1) is extremal in each such class for a common value of $\alpha$ that is approximately equal to 0.5343598 . Moreover, since the classes are dense in the space of all hyperbolically convex functions, this $f_{\alpha}$ must be extremal for all hyperbolically convex functions. Indeed, up to disc automorphisms, $f_{\alpha}$ can be the only extremal function which maps onto a finite-sided hyperbolically convex polygon. However, this does not rule out the possibility, that some function onto a non-polygonal region could have the same Schwarz norm as $f_{\alpha}$. Thus our methods do not guarantee uniqueness, but we conjecture that the extremal is in fact unique.

In Section 2 we develop background material on hyperbolic convexity and the Schwarzian derivative and discuss more of the history of the problem. In Section 3 we first show by an approximation argument that the problem can be reduced to studying the value of the Schwarzian at the origin for those functions which map onto finite-sided polygons.

We then give a generalization of the Julia Variational Formula that we will use in Sections 4 and 5 to develop two variations which preserve hyperbolic convexity. In Section 6 we apply these variations to show the extremal polygon has at most two proper sides. Finally, in Section 7 we employ inequalities on elliptic integrals to verify that the two-sided domain conjectured by Mejía and Pommerenke $[10,12,14]$ is indeed extremal.

We would like to thank the Phillip Brown, the referee and the editor for their very valuable suggestions.

\section{Hyperbolic Convexity and Schwarzians}

\subsection{Hyperbolic Geometry}

The unit disc $\mathbb{D}$ equipped with the metric

$$
d_{h}(z, w)=\inf \left\{\int_{\gamma} \frac{1}{1-|z|^{2}}|d z|: \gamma \text { is a rectifiable curve joining } z \text { and } w\right\}
$$

forms a model for the hyperbolic plane [5]. Notice that the Poincaré density

$$
\eta_{\mathbb{D}}(z)=\frac{1}{1-|z|^{2}}
$$

goes to infinity as $z$ moves toward the boundary of the disc. Consequently, integrating $\eta_{\mathbb{D}}$ over curves near the boundary produces large values of the integral. If $z$ and $w$ do not lie on a ray through the origin, then the euclidean line segment joining them will produce a larger integral than a curve which bends away from the boundary. In fact, the infimum will be achieved by an arc of a circle perpendicular to $\partial \mathbb{D}$. Such curves are hyperbolic geodesics. Since disc automorphisms

$$
M(z)=e^{i \theta} \frac{z-a}{1-\bar{a} z},
$$


4 Roger W. BARNARD, LEAH COLE, KENT PEARCE, AND G. BROCK WILLIAMS

where $\theta \in[0,2 \pi)$ and $a \in \mathbb{D}$, preserve circles orthogonal to $\partial \mathbb{D}$, they are precisely the isometries of $\mathbb{D}$.

Any region $G$ conformally equivalent to $\mathbb{D}$ also carries a hyperbolic metric defined in the same manner using the density

$$
\eta_{G}(z)=\frac{\left|f^{\prime}(z)\right|}{1-|f(z)|^{2}},
$$

where $f$ is a conformal map of $G$ onto $\mathbb{D}$. Notice that it doesn't matter which map $f$ is chosen as any two such maps must differ by a disc automorphism.

\subsection{Convexity}

The euclidean notion of convexity generalizes to hyperbolic regions in an obvious manner.

DEFINITION 2.1. A region $\Omega \subset \mathbb{D}$ is hyperbolically convex if for any two points $z, w \in \Omega$, the hyperbolic geodesic segment joining $z$ and $w$ lies completely in $\Omega$.

Notice that since the disc automorphisms are the isometries of the hyperbolic plane, the image $M(\Omega)$ of $\Omega$ under a disc automorphism $M$ is hyperbolically convex if and only if $\Omega$ is. The fundamental domains of discrete groups of disc automorphisms provide a great many useful examples of hyperbolically convex domains. See Beardon [5] for an extensive discussion of these regions.

We will call a hyperbolically convex region $\Omega$ bounded by a finite number of either geodesic arcs lying inside $\mathbb{D}$ or arcs of $\partial \mathbb{D}$ a hyperbolically convex polygon. We call the bounding geodesic arcs proper sides and the arcs of $\partial \mathbb{D}$ improper sides. For $n \geq 0$, we let

$$
\begin{aligned}
K_{n}= & \{\text { hyperbolically convex polygons containing } 0 \\
& \text { and having at most } n \text { proper sides }\} \cup\{0\} .
\end{aligned}
$$

DEFINITION 2.2. A conformal map $f: \mathbb{D} \rightarrow \Omega$ is called a hyperbolically convex function if its range is hyperbolically convex. We let $H$ denote the class of all hyperbolically convex functions that fix the origin and let $H_{n}$ denote the subset of functions whose range is in $K_{n}$.

\subsection{Schwarzians}

Much of the geometric behavior of an analytic function is described by its Schwarzian derivative $[6,7]$.

Definition 2.3. The Schwarzian derivative (or just "Schwarzian") of an analytic function $f$ is

$$
S_{f}=\left(\frac{f^{\prime \prime}}{f^{\prime}}\right)^{\prime}-\frac{1}{2}\left(\frac{f^{\prime \prime}}{f^{\prime}}\right)^{2}
$$

Proposition 2.4. The Schwarzian of an analytic function is identically 0 if and only if it is a Möbius transformation. Moreover, the Schwarzian satisfies the 
chain rule

$$
S_{f \circ g}=\left(S_{f} \circ g\right)\left(g^{\prime}\right)^{2}+S_{g}
$$

Thus, if $M$ is Möbius, then

$$
S_{M \circ g}=S_{g}
$$

and

$$
S_{f \circ M}=\left(S_{f} \circ M\right)\left(M^{\prime}\right)^{2} .
$$

Hence the Schwarzian is unchanged by post-composition with a Möbius transformation, but pre-composition produces an extra quadratic factor.

DeFINITION 2.5. Let $f$ be defined on a simply connected region $G \subset \mathbb{C}$. The Schwarz norm of $f$ is given by

$$
\left\|S_{f}\right\|_{G}=\sup _{z \in G} \eta_{G}^{-2}(z)\left|S_{f}(z)\right| .
$$

By taking into account the density of the hyperbolic metric, the Schwarz norm is completely Möbius invariant. It is easy to show for any Möbius $M$ that

$$
\eta_{M^{-1}(G)}(z)=\eta_{G}(M(z))\left|M^{\prime}(z)\right|
$$

and thus

$$
\frac{\left|S_{f \circ M}(z)\right|}{\eta_{M^{-1}(G)}^{2}(z)}=\frac{\left|S_{f}(M(z))\right|\left|M^{\prime}(z)\right|^{2}}{\eta_{G}^{2}(M(z))\left|M^{\prime}(z)\right|^{2}}=\frac{\left|S_{f}(w)\right|}{\eta_{G}^{2}(w)} .
$$

where $w=M(z)$. Thus

$$
\left\|S_{f}\right\|_{G}=\left\|S_{f \circ M}\right\|_{M^{-1}(G)}
$$

and

$$
\left\|S_{f}\right\|_{G}=\left\|S_{M \circ f}\right\|_{G}
$$

In particular, notice that $\left\|S_{f}\right\|_{\mathbb{D}}$ is unchanged by disc automorphisms.

\subsection{Computing the Schwarz Norm of a Function}

Because of the Möbius invariance of the Schwarz norm, we can change the domain of a function so that the hyperbolic density or the expression of the function itself is simpler.

EXAMPLE 2.6. Consider the function

$$
g(z)=\frac{\sqrt{z}-1}{\sqrt{z}+1}
$$

from the upper half plane $\mathbb{H}$ onto the upper half-disc $\mathbb{D}^{+}=\{z \in \mathbb{D}: \operatorname{Im} z>0\}$. Notice that any hyperbolically convex domain bounded by exactly one proper side can be mapped onto $\mathbb{D}^{+}$by a disc automorphism. Moreover, $g$ could have been defined on $\mathbb{D}$ by precomposition with a Möbius transformation; however, that would not change the Schwarz norm. Consequently, the Schwarz norm for any hyperbolically convex function whose image is bounded by exactly one proper side must be equal to $\left\|S_{g}\right\|_{\mathbb{H}}$.

Next notice that $g$ itself is the composition of $h(z)=\sqrt{z}$ and another Möbius 
6 Roger W. BARNARd, LEAH COLE, KEnt PEARCE, AND G. BRock Williams

transformation. Thus $\left\|S_{g}\right\|_{\mathbb{H}}=\left\|S_{h}\right\|_{\mathbb{H}}$. It is now easy to compute

$$
S_{h}(z)=\frac{3}{8 z^{2}}
$$

The hyperbolic density on $\mathbb{H}$ has an especially useful representation

$$
\eta_{\mathbb{H}}(z)=\frac{1}{2 \operatorname{Im} z} .
$$

Since both $\eta_{\mathbb{H}}$ and $S_{h}$ are invariant under translations of the form $x+i y \rightarrow(x+$ a) $+i y$,

$$
\left\|S_{h}\right\|_{\mathbb{H}}=\sup _{z \in \mathbb{H}} \eta^{-2}(z)\left|S_{h}(z)\right|=\sup _{y>0} 4 y^{2}\left|\frac{3}{8(i y)^{2}}\right|=\frac{3}{2}
$$

\subsection{Geometry of the Schwarzian}

Since $\left\|S_{f}\right\|_{\mathbb{D}}=0$ if and only if $f$ is Möbius, we can view $\left\|S_{f}\right\|_{\mathbb{D}}$ as measuring how close $f$ is to being a Möbius transformation. Since any Möbius transformation would send $\mathbb{D}$ to another disc or half plane, $\left\|S_{f}\right\|_{\mathbb{D}}$ also measures the amount of deformation between $f(\mathbb{D})$ and a disc. This notion was formalized by Lehto [7] to produce a pseudometric between regions conformally equivalent to a disc.

There are a number of results that show that if $\left\|S_{f}\right\|_{\mathbb{D}}$ is small, then $f(\mathbb{D})$ possesses disc-like properties. The two most important for our purposes are due to Nehari $[16,18]$.

THEOREM 2.7. If $\left\|S_{f}\right\|_{\mathbb{D}}<2$, then $f$ is univalent and $f(\mathbb{D})$ is a quasidisc. Moreover, if $f$ is univalent, then $\left\|S_{f}\right\|_{\mathbb{D}} \leq 6$.

THEOREM 2.8. If $f(\mathbb{D})$ is convex (in the euclidean sense), then $\left\|S_{f}\right\|_{\mathbb{D}} \leq 2$, with equality if and only if $f(\mathbb{D})$ is an infinite strip.

Mejía and Pommerenke [11] proved a similar result for spherically convex regions.

THEOREM 2.9. If $f(\mathbb{D})$ is spherically convex, then

$$
\left\|S_{f}\right\|_{\mathbb{D}} \leq 2\left(1-\sigma(f)^{2}\right),
$$

where

$$
\sigma(f)=\max _{z \in \mathbb{D}}\left(1-|z|^{2}\right) \frac{\left|f^{\prime}\right|}{1+|f|^{2}} .
$$

For a fixed value of $\sigma(f)$, this maximum value of $\left\|S_{f}\right\|_{\mathbb{D}}$ is achieved by a map of the form $f_{\phi}(z)=i \tanh \left(\frac{2 \phi}{\pi} \log \left(\frac{1+z}{1-z}\right)\right)$ which takes $\mathbb{D}$ onto a "spherical strip," that is, a lune bounded by great circles through $\pm i$ and making an angle $2 \phi$ with the imaginary axis.

Thus, convex and spherically convex regions cannot be deformed too far from being a disc in the sense of the Lehto pseudometric, and the regions with the greatest amount of deformation are strips. It has been conjectured by Mejía and Pommerenke $[10,12,14]$ that the same must hold for hyperbolically convex regions. In particular, Mejía and Pommerenke conjectured that the maximum value of 

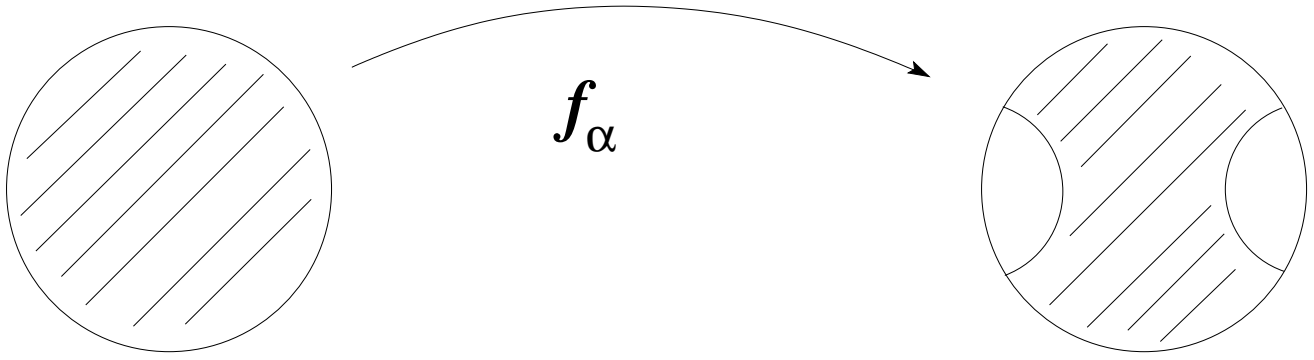

FIGURE 2. The hyperbolically convex extremal function for maximizing the Schwarz norm. The extremal domain consists of an odd symmetric polygon bounded by two proper geodesic sides - a "hyperbolic strip."

$\left\|S_{f}\right\|_{\mathbb{D}}$ over all hyperbolically convex maps $f$ is achieved by

$$
f_{\alpha}(z)=\tan \left(\alpha \int_{0}^{z}\left(1-2 \xi^{2} \cos 2 \theta+\xi^{4}\right)^{-1 / 2} d \xi\right), \quad \alpha=\frac{\pi}{2 K(\cos \theta)},
$$

where $K$ is the elliptic integral of the first kind. The range of $f_{\alpha}$ is a "hyperbolic strip" bounded by two geodesics through $\pm i \tanh \left(\frac{\pi K(\sin \theta)}{4 K(\cos \theta)}\right)$. See Figure 2 .

\section{Preliminary Simplifications}

\subsection{Reduction to Polygonal Domains}

As a first step toward verifying Mejía and Pommerenke's conjecture, we first reduce the problem to hyperbolically convex polygons.

LEMma 3.1. To determine the extremal value of $\left\|S_{f}\right\|_{\mathbb{D}}$ over $H$, it suffices to determine the extremal value over each $H_{n}$. Moreover, each $H_{n}$ is compact.

Proof.

From its definition as a supremum, it follows that the Schwarz norm is a lower semi-continuous functional on $H$; that is, for any point $f \in H$, if $\left\{f_{n}\right\}$ is a sequence in $H$ which converges to $f$ (locally uniformly on compacta), then $\lim \inf \left\|S_{f_{n}}\right\|_{\mathbb{D}} \geq$ $\left\|S_{f}\right\|_{\mathbb{D}}$. To see this, let $\epsilon>0$. Then, for any compact subset $K$ of $\mathbb{D}$, there exists an index $N$ such that for $n \geq N$ we have $\left\|S_{\left.f_{n}\right|_{\text {int } K}}\right\|_{\mathbb{D}} \geq\left\|S_{\left.f\right|_{\text {int } K}}\right\|_{\mathbb{D}}-\epsilon$. Hence, for $n \geq N$ we have $\left\|S_{f_{n}}\right\|_{\mathbb{D}} \geq\left\|S_{\left.f_{n}\right|_{\text {int } K}}\right\|_{\mathbb{D}} \geq\left\|S_{\left.f\right|_{\text {int } K}}\right\|_{\mathbb{D}}-\epsilon$. Hence, $\liminf \left\|S_{f_{n}}\right\|_{\mathbb{D}} \geq$ $\left\|S_{f_{\text {int } K}}\right\|_{\mathbb{D}}-\epsilon$. Since $K$ was abritrary we must have, liminf $\left\|S_{f_{n}}\right\|_{\mathbb{D}} \geq\left\|S_{f}\right\|_{\mathbb{D}}-\epsilon$. Since $\epsilon$ was arbitrary, we have $\liminf \left\|S_{f_{n}}\right\|_{\mathbb{D}} \geq\left\|S_{f}\right\|_{\mathbb{D}}$.

For a fixed $n$, a sequence $D_{k}$ of hyperbolically convex polygonal domains with at most $n$ (proper) sides can converge in the sense of Carathéodory [20] only to another polygonal domain $D_{0}$, where $D_{0}$ has at most $n$ (proper) sides. This follows because if $D_{0}$ had more than $n$ proper sides, then (from the boundary behavior) the Carathéodory convergence [20] would imply, for $k$ sufficiently large, that $D_{k}$ would have at least $n+1$ proper sides. From Carathéodory 's Convergence Theorem, this carries over to hyperbolically convex functions in $H_{n}$. Thus, $H_{n}$ is compact.

On the other hand, the boundary $\Gamma$ of a hyperbolically convex domain $D$ can be approximated by geodesics lying in $\overline{\mathbb{D}}$ by connecting points on $\Gamma$ sufficiently 
8 Roger W. BARNARD, LEAH COLE, KENT PEARCE, AND G. BROCK WILLIAMS

close together with geodesics lying in $\overline{\mathbb{D}}$. It follows that we can find a sequence of hyperbolically convex polygons that converge, in the sense of Carathéodory, to $D$. Hence, the mapping functions onto these polygons converge locally uniformly to the map onto $D$. As a result, $\cup_{n} H_{n}$ is dense in $H$.

Thus any extremal function in $H$ can be approximated by functions in $H_{n}$. As a result, it suffices to determine the supremum of $\left\|S_{f}\right\|_{\mathbb{D}}$ in each $H_{n}$.

\subsection{Reduction to $S_{f}(0)$}

Recall that the Schwarz norm was defined by taking the supremum of $\mid S_{f}\left(z \mid\left(1-|z|^{2}\right)^{2}\right.$ over all points $z \in \mathbb{D}$. Since $\mid S_{f}\left(z \mid\left(1-|z|^{2}\right)^{2}\right.$ is invariant under disc automorphisms, we need only consider $\left|S_{f}(0)\right|$.

LEMMA 3.2. For each $n \geq 2$,

$$
\sup _{f \in H_{n}}\left\|S_{f}\right\|_{\mathbb{D}}=\max _{f \in H_{n}}\left|S_{f}(0)\right| \text {. }
$$

Proof. Recall that every $f \in H_{n}$ is conjugate by a Möbius transformation to a map $g: \mathbb{H} \rightarrow \mathbb{H}$ which is hyperbolically convex in the upper half plane model. Suppose $g$ maps $\mathbb{H}$ onto a curvilinear polygon with angles $\alpha_{1} \pi, \alpha_{2} \pi, \ldots, \alpha_{n} \pi$, and the preimages of the vertices are $a_{1}, a_{2}, \ldots, a_{n} \in \mathbb{R}$. Then the Schwarzian of $g$ has the following very useful representation (see Nehari [17])

$$
S_{g}(z)=\frac{1}{2} \sum_{k=1}^{n} \frac{1-\alpha_{k}^{2}}{\left(z-a_{k}\right)^{2}}+\sum_{k=1}^{n} \frac{\beta_{k}}{z-a_{k}},
$$

where $\beta_{1}, \beta_{2}, \ldots, \beta_{n}$ are real constants determined by the angles. Thus

$$
\left\|S_{g}\right\|_{\mathbb{H}}=\sup _{z \in \mathbb{H}} 4(\operatorname{Im} z)^{2}\left|\frac{1}{2} \sum_{k=1}^{n} \frac{1-\alpha_{k}^{2}}{\left(z-a_{k}\right)^{2}}+\sum_{k=1}^{n} \frac{\beta_{k}}{z-a_{k}}\right| .
$$

Notice however, that

$$
\lim _{\substack{z \rightarrow a \in \mathbb{R} \\ a \neq a_{k}}} 4(\operatorname{Im} z)^{2}\left|\frac{1}{2} \sum_{k=1}^{n} \frac{1-\alpha_{k}^{2}}{\left(z-a_{k}\right)^{2}}+\sum_{k=1}^{n} \frac{\beta_{k}}{z-a_{k}}\right|=0
$$

and

$$
\lim _{z \rightarrow a_{k}} 4(\operatorname{Im} z)^{2}\left|\frac{1}{2} \sum_{k=1}^{n} \frac{1-\alpha_{k}^{2}}{\left(z-a_{k}\right)^{2}}+\sum_{k=1}^{n} \frac{\beta_{k}}{z-a_{k}}\right|=2\left(1-\alpha_{k}^{2}\right)<2 .
$$

Applying the relationships between $\alpha_{i}$ and $\beta_{i}$ given by Nehari [17], we can similarly show

$$
\lim _{z \rightarrow \infty} 4(\operatorname{Im} z)^{2}\left|\frac{1}{2} \sum_{k=1}^{n} \frac{1-\alpha_{k}^{2}}{\left(z-a_{k}\right)^{2}}+\sum_{k=1}^{n} \frac{\beta_{k}}{z-a_{k}}\right|
$$

is also bounded by 2 .

In view of the invariance of the Schwarz norm under Möbius transformations, the same bounds must be true for mappings of $\mathbb{D}$ onto hyperbolic polygons in $\mathbb{D}$. It is not difficult [10] to construct mappings of $\mathbb{D}$ onto hyperbolic polygons with 
$\left\|S_{f}\right\|_{\mathbb{D}} \geq 2$. Thus if $f$ maps $\mathbb{D}$ onto a hyperbolic polygon and $f$ is extremal for maximizing the Schwarz norm, then the supremum in the definition of the Schwarz norm must in fact be attained at some interior point.

Suppose $f$ is extremal in $H_{n}$ for maximizing the Schwarz norm and

$$
\max _{z \in \mathbb{D}}\left(1-|z|^{2}\right)^{2}\left|S_{f}(z)\right|=\left(1-\left|z_{0}\right|^{2}\right)^{2}\left|S_{f}\left(z_{0}\right)\right| .
$$

Since $\left(1-|z|^{2}\right)^{2}\left|S_{f}(z)\right|$ is invariant under Möbius transformations, precomposing $f$ with a disc automorphism which sends zero to $z_{0}$ will produce a map $f_{0}$ which has the same Schwarz norm as $f$ but for which $\left\|S_{f_{0}}\right\|=\left|S_{f_{0}}(0)\right|$.

Notice by precomposing $f$ with a disc automorphism, our new map $f_{0}$ need not fix 0 and thus need not remain in $H_{n}$. However, by postcomposing $f_{0}$ with a disc automorphism which sends $f_{0}(0)$ to 0 and then rotates so that $S_{f_{0}}(0)$ is real, we can constuct an extremal function $F \in H_{n}$ with

$$
\left\|S_{f}\right\|_{\mathbb{D}}=\left\|S_{F}\right\|_{\mathbb{D}}=S_{F}(0) .
$$

Since the functional $S_{f}(0)$ is continuous on $H_{n}$ and $H_{n}$ is compact, then

$$
\sup _{f \in H_{n}}\left\|S_{f}\right\|_{\mathbb{D}}=\sup _{f \in H_{n}} S_{f}(0)=\max _{f \in H_{n}} S_{f}(0) .
$$

\section{Variational Techniques}

\subsection{The Julia Variation and Extensions}

Let $\Omega$ be a region bounded by a piecewise analytic curve $\Gamma$ and $\phi(w)$ be a positive piecewise $C^{1}$ function on $\Gamma$, vanishing where $\Gamma$ is not analytic. Denote the outward pointing unit normal vector at each point $w$ where $\Gamma$ is smooth by $n(w)$. For $\epsilon$ near 0 , construct a new curve

$$
\Gamma_{\epsilon}=\{w+\epsilon \phi(w) n(w): w \in \Gamma\}
$$

and let $\Omega_{\epsilon}$ be the new region bounded by $\Gamma_{\epsilon}$.

For our purposes, we may assume that $\Omega$ is simply connected. Thus if $\epsilon$ is sufficiently small and $\epsilon>0$, then $\Gamma$ is "pushed outside" the domain, while if $\epsilon<0$, then $\Gamma$ is "pushed inside" the domain. With the above notation, we state Julia's variational formula.

Julia Variational Formula. Let $f$ be a conformal map from $\mathbb{D}$ onto $\Omega$ with $f(0)=0$, and suppose that $f$ has a continuous extension to $\partial \mathbb{D}$, which we also denote by $f$. Then for $\epsilon$ sufficiently small, a similarly normalized conformal map $f_{\epsilon}$ from $\mathbb{D}$ onto $\Omega_{\epsilon}$, with $f_{\epsilon}(0)=0$, is given by

$$
f_{\epsilon}(z)=f(z)+\frac{\epsilon z f^{\prime}(z)}{2 \pi} \int_{\partial \mathbb{D}} \frac{1+\xi z}{1-\xi z} d \Psi+E(\epsilon, z),
$$

where $d \Psi=\frac{\phi(f(\xi))}{\left|f^{\prime}(\xi)\right|} d \theta$ and $\xi=e^{i \theta}$ and $E(\epsilon, z)$ is o $(\epsilon)$ for $z$ on compact subsets of $\mathbb{D}$ and is continuously differentiable in $\epsilon$ for each fixed $z \in \mathbb{D}$. 


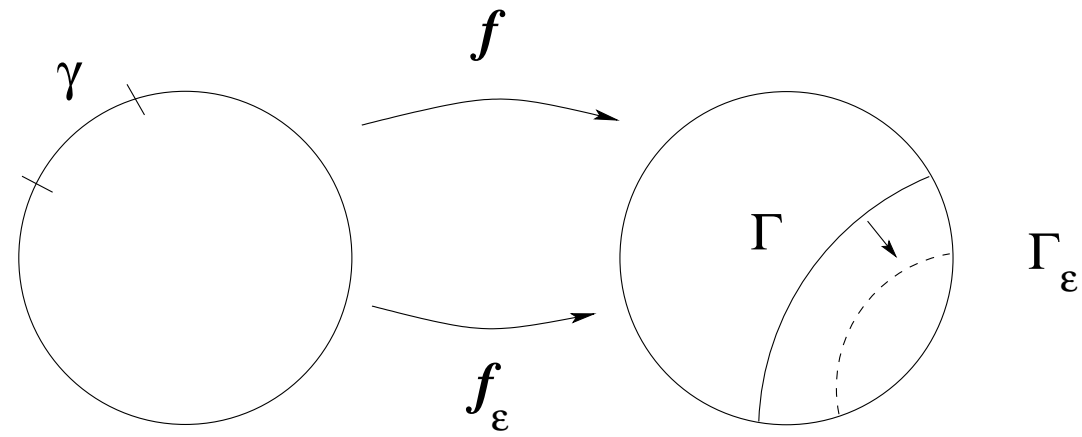

FiguRE 3. The variation produced by "pushing out" a side.

Notice the restriction that $\phi$ vanish at the points of non-smoothness of $\partial \Omega$ was a strong one. It implies, for example, that while we could vary the (proper) sides of a hyperbolic polygon, there is a difficulty at the vertices. However, it follows from the work of the first author and J. Lewis [3] that such an extended version of the Julia variation is possible (except at internal cusps, ie., where two proper sides meet at an angle of measure $2 \pi$ ) which allows $f^{\prime}$ to vanish at the corners. Moreover, they showed that the resulting function will agree with the Julia variational formula on compact subsets up to $o(\epsilon)$ terms. This will allow us to create variations which avoid any problems at the corners and preserve hyperbolic convexity.

\subsection{Two Variations}

We next describe in detail two variations for functions in $H_{n}$ which preserve hyperbolic convexity. First, if $f$ maps onto a hyperbolically convex polygon $\Omega$ with a proper side $\Gamma$, we can "push" $\Gamma$ to a nearby geodesic $\Gamma_{\epsilon}$ in such a way that the varied function $f_{\epsilon}$ will still be hyperbolically convex. See Figure 3.

LEMma 4.1. Suppose $f \in H_{n}$ and $f$ is not constant nor the identity map. If $\Gamma=f(\gamma)$ is a proper side of $\Omega=f(\mathbb{D})$, then for $\epsilon$ sufficiently small there exists a variation $f_{\epsilon} \in H_{n}$ which "pushes" $\Gamma$ to a nearby geodesic $\Gamma_{\epsilon}$, where $\Gamma_{\epsilon} \rightarrow \Gamma$ as $\epsilon \rightarrow 0$. This variation, $f_{\epsilon}$, agrees with the Julia Variational Formula on compact subsets up to o( $\epsilon)$ terms.

\section{Proof.}

Let $\Gamma$ be a (proper) side of the boundary of $\Omega$ which lies on a circle $\Lambda$. Without loss of generality we can assume that $\Omega$ has been rotated so that the center of $\Lambda$ lies on the positive real-axis. To define our variation first map $\Omega$ into the right halfplane under the map $h(z)=\frac{1+z}{1-z}$ and let $\Upsilon=h(\Gamma)$ and $\Delta=h(\Omega)$. Let $g=h \circ f$. We note that $\Delta=g(\mathbb{D})$.

Under $h$, the geodesics in $\mathbb{D}$ map to arcs in the right half-plane which lie on circles whose centers are on the imaginary axis. We note that $h(\Lambda)$ is a circle centered at the origin. Let the arc $\widehat{A B}$ denote $\Upsilon$ on the circle $h(\Lambda)$ and let $r_{\Lambda}$ be the radius of $h(\Lambda)$.

Now, considering the case where $\epsilon>0$, i.e., the case where we "push out" a (proper) side of the domain by a euclidean distance $\epsilon$ and thus enlarge the domain. 


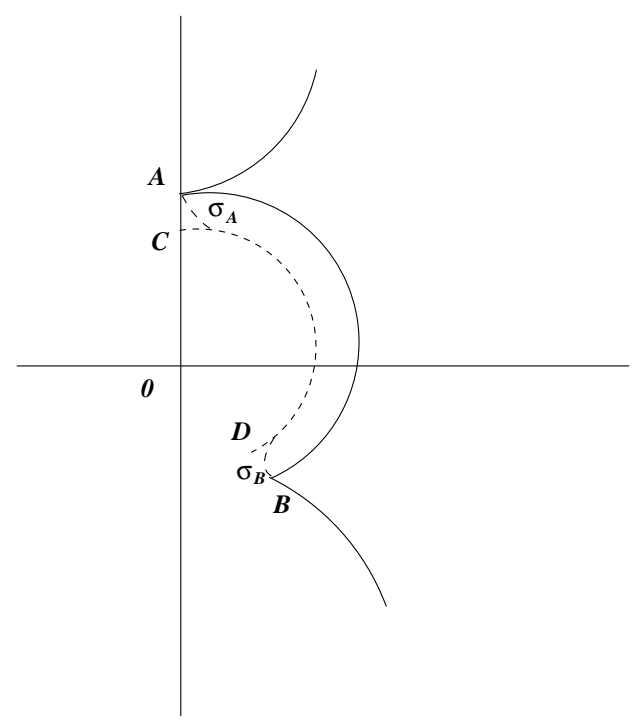

FIGURE 4. The geodesics in the right half plane produced by our variation.

Let the circle $\chi$ of radius $r=r_{\Lambda}-\epsilon$ be obtained by multiplying $h(\Lambda)$ by $1-\epsilon / r_{\Lambda}$ and let $\widehat{C D}$ be the arc of $\chi$ which connects the extensions of the circles defined by the images of the $\Gamma$ 's adjacent geodesics under $h$, when $A$ and $B$ are in the right-half plane. If $A$ or $B$ is on the imaginary axis, then $C$ or $D$ will be the point on the imaginary axis where $\chi$ intersects the imaginary axis, resp. In this case, the interval $[A, C]$ or $[D, B]$ will be added to the boundary of our varied domain, resp.

Now recall in the original Julia variation formula, the function $\phi$ must be 0 at the end points of $\widehat{A B}$. Thus at each end of $\widehat{A B}$, we construct approximating smooth curves $\sigma_{A}$ and $\sigma_{B}$ which connect $\widehat{A B}$ to $\widehat{C D}$ such that $\sigma_{A}$ and $\sigma_{B}$ are orthogonal to $\widehat{A B}$ at the endpoints $A$ and $B$ and $\sigma_{A}$ and $\sigma_{B}$ intersect $\widehat{C D}$ tangentially at points $C^{\prime}$ and $D^{\prime}$, resp. They can be chosen to be within $\epsilon^{2}$ of the arc they are approximating. See Figure 4.

Now for $w \in \widehat{A B}$ let $n(w)$ be the outward unit normal to $g(\Lambda)$ and define $\phi(w)=$ $\left|w^{*}-w\right|$ where $w^{*}$ is the nearest point on $\sigma_{A} \cup \widehat{C^{\prime} D^{\prime}} \cup \sigma_{B}$ along the normal $n(w)$. Let the domain $\Delta_{\epsilon}^{*}$ be the variation of $\Delta$ where the arc $\widehat{A B}$ on the boundary of $\Delta$ has been replaced by $\sigma_{A} \cup \widehat{C^{\prime} D^{\prime}} \cup \sigma_{B}$. To obtain a variation of $\Delta$ which preserves hyperbolic convexity, let the domain $\Delta_{\epsilon}$ be the variation of $\Delta$ where the arc $\widehat{A B}$ has been replaced by $\widehat{C D}$ and connecting intervals, if necessary. Let $g_{\epsilon}^{*}$ and $g_{\epsilon}$ be the corresponding mapping functions onto $\Delta_{\epsilon}^{*}$ and $\Delta_{\epsilon}$, resp. Let $\phi(w)=0$ for $w \in \partial \Delta \backslash \widehat{A B}$. Then, by the Julia Variational Formula, $g_{\epsilon}^{*}$ satisfies

$$
g_{\epsilon}^{*}(z)=g(z)+\frac{\epsilon z g^{\prime}(z)}{2 \pi} \int_{\partial \mathbb{D}} \frac{1+\xi z}{1-\xi z} d \Psi+o(\epsilon),
$$

on compact subsets, where $d \Psi=\frac{\phi(g(\xi))}{\left|g^{\prime}(\xi)\right|} d \theta$ and $\xi=e^{i \theta}$. Rewriting $g$ as $h \circ f$, we have 
12 Roger W. BARNARD, LEAH COLE, KENT PEARCE, AND G. BROCK WILLIAMS

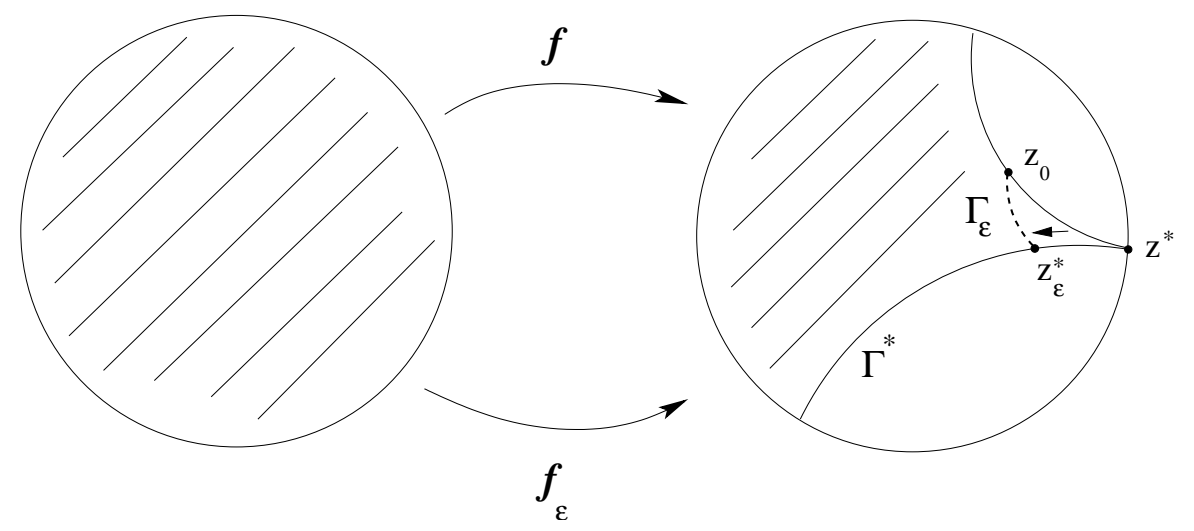

FIGURE 5. The variation produced by pushing in one end of a side.

$$
g_{\epsilon}^{*}(z)=h(f(z))+\frac{\epsilon z h^{\prime}(f(z)) f^{\prime}(z)}{2 \pi} \int_{\partial \mathbb{D}} \frac{1+\xi z}{1-\xi z} d \Psi+o(\epsilon),
$$

on compact subsets, where $d \Psi=\frac{\phi(h(f(\xi)))}{\left|h^{\prime} f((\xi)) f^{\prime}(\xi)\right|} d \theta$ and $\xi=e^{i \theta}$.

The function $g_{\epsilon}^{*}$ was constructed so that it satisfies the requirements for representation by the Julia Variational Formula. The function $g_{\epsilon}$ was constructed so that image, $g_{\epsilon}(\mathbb{D})$, was hyperbolically convex. The arguments used in [3], pp 348-356, show that $g_{\epsilon}^{*}-g_{\epsilon}=o(\epsilon)$ on compact subsets. Then, defining $f_{\epsilon}=h^{-1} \circ g_{\epsilon} \equiv \frac{g_{\epsilon}-1}{g_{\epsilon}+1}$, a straightforward argument shows that

$$
f_{\epsilon}(z)=f(z)+\frac{\epsilon z f^{\prime}(z)}{2 \pi} \int_{\partial \mathbb{D}} \frac{1+\xi z}{1-\xi z} d \Psi+o(\epsilon)
$$

on compact subsets, where $\Psi$ is a real measure on $\partial \mathbb{D}$ and $\xi=e^{i \theta}$. Thus, $f_{\epsilon}$ maps $\mathbb{D}$ onto a hyperbolically convex domain, i.e., $f_{\epsilon} \in H_{n}$ for $\epsilon$ sufficiently small.

A similar variation can be defined for $\epsilon<0$ that "pushes in" the (proper) side $\Gamma$ to a nearby geodesic.

If $\Gamma$ intersects another side $\Gamma^{*}$ at $z^{*}$, and $z_{0} \in \Gamma$, then we can vary $f$ so as to replace the portion of $\Gamma$ between $z_{0}$ and $z^{*}$ with a new geodesic between $z_{0}$ and some $z_{\epsilon}^{*} \in \Gamma^{*}$. See Figure 5. A similar argument to the above then gives:

LEMMA 4.2. Suppose $f \in H_{n}$ and $f$ is not constant nor the identity map, and $\Gamma=f(\gamma)$ is a proper side of $f(\mathbb{D})$ meeting a side $\Gamma^{*}$. Then, there exists a variation $f_{\epsilon} \in H_{n+1}$ which adds a proper side to $f(\mathbb{D})$ by pushing one end of $\Gamma$ to a nearby side $\Gamma_{\epsilon}$. That is, $f_{\epsilon}(\mathbb{D})$ is a hyperbolic polygon whose sides are the same as those of $f$, except that one end of $\Gamma$ has been replaced by $\Gamma_{\epsilon}$ and $\Gamma^{*}$ has been shortened. Moreover, $f_{\epsilon}$ agrees with the Julia Variational Formula on compact subsets up to $o(\epsilon)$ terms.

REMARK 4.3. Notice that in order to maintain hyperbolic convexity, we can only push in the "end" of a side, that is, a subarc that ends at a vertex of the 
polygon. However, we can choose this subarc to be as long, or more importantly, as short, as we wish.

\section{The Effect of the Variations on the Schwarzian}

Recall from Lemma 3.2, we can assume our extremal function satisfies $\left\|S_{f}\right\|_{\mathbb{D}}=$ $S_{f}(0)$. In this case, the Schwarz norm has a simple representation in terms of power series coefficients $[7,10]$.

Proposition 5.1. If $f=\alpha\left(z+a_{2} z^{2}+a_{3} z^{3}+\ldots\right)$, then

$$
S_{f}(0)=6\left(a_{3}-a_{2}^{2}\right) \text {. }
$$

Proof.

Since $f^{\prime}(0)=\alpha, f^{\prime \prime}(0)=2 \alpha a_{2}$, and $f^{\prime \prime \prime}(0)=6 \alpha a_{3}$,

$$
\begin{aligned}
\left.\frac{d}{d z} \frac{f^{\prime \prime}(z)}{f^{\prime}(z)}\right|_{z=0} & =\frac{f^{\prime}(0) f^{\prime \prime \prime}(0)-\left(f^{\prime \prime}(0)\right)^{2}}{\left(f^{\prime}(0)\right)^{2}} \\
& =\frac{6 \alpha^{2} a_{3}-4 \alpha^{2} a_{2}^{2}}{\alpha^{2}} \\
& =6 a_{3}-4 a_{2}^{2} .
\end{aligned}
$$

Also,

$$
\frac{1}{2}\left(\frac{f^{\prime \prime}(0)}{f^{\prime}(0)}\right)^{2}=\frac{1}{2} \frac{4 \alpha^{2} a_{2}^{2}}{\alpha^{2}}=2 a_{2}^{2}
$$

Consequently,

$$
S_{f}(0)=\left(\left(\frac{f^{\prime \prime}}{f^{\prime}}\right)^{\prime}-\frac{1}{2}\left(\frac{f^{\prime \prime}}{f^{\prime}}\right)^{2}\right)=6\left(a_{3}-a_{2}^{2}\right) .
$$

Now applying the representation for the variations provided by the Julia Formula, we can calculate to first order in $\epsilon$ the Schwarzian of our varied functions.

LEMmA 5.2. If $f(z)=\alpha\left(z+a_{2} z^{2}+a_{3} z^{3}+\ldots\right) \in H_{n}$ and either of the variations described in Lemma 4.1 and Lemma 4.2 are applied to $f$, then the new function $f_{\epsilon}(z)$ has $S_{f_{\epsilon}}(0)$ given by

$6\left(\left(a_{3}+\frac{\epsilon}{2 \pi} \int_{\Gamma}\left(3 a_{3}+4 a_{2} \xi+2 \xi^{2}\right) d \Psi\right)-\left(a_{2}+\frac{\epsilon}{2 \pi} \int_{\Gamma}\left(2 a_{2}+2 \xi\right) d \Psi\right)^{2}\right)+o(\epsilon)$.

Proof.

Let $\gamma$ be the preimage in $\partial \mathbb{D}$ of the portion of the boundary we are varying. Expanding $\frac{1+\xi z}{1-\xi z}$ as a series, we obtain on compact subsets

$$
\begin{aligned}
f_{\epsilon}(z) & =f(z)+\frac{\epsilon z f^{\prime}(z)}{2 \pi} \int_{\gamma} \frac{1+\xi z}{1-\xi z} d \Psi+o(\epsilon) \\
& =f(z)+\frac{\epsilon z f^{\prime}(z)}{2 \pi} \int_{\gamma}\left(1+2 \xi z+2 \xi^{2} z^{2}+2 \xi^{3} z^{2}+\ldots\right) d \Psi+o(\epsilon) .
\end{aligned}
$$


14 Roger W. BARNARD, LEAH COLE, KENT PEARCE, AND G. BROCK WILLIAMS

Now since $z f^{\prime}(z)=\alpha\left(z+2 a_{2} z^{2}+3 a_{3} z^{3}+\ldots\right)$, we have

$$
f_{\epsilon}(z)=f(z)+\frac{\epsilon \alpha}{2 \pi} \int_{\gamma}\left[z+2\left(a_{2}+\xi\right) z^{2}+\left(3 a_{3}+4 a_{2} \xi+2 \xi^{2}\right) z^{3}+\ldots\right] d \Psi+o(\epsilon)
$$

on compact subsets.

Finally, gathering the powers of $z$, we arrive at

$$
\begin{aligned}
f_{\epsilon}(z)=\alpha & \left(1+\frac{\epsilon}{2 \pi} \int_{\gamma} d \Psi\right) z+\left(a_{2}+\frac{\epsilon}{2 \pi} \int_{\gamma} 2\left(a_{2}+\xi\right) d \Psi\right) z^{2} \\
& \left.+\left(a_{3}+\frac{\epsilon}{2 \pi} \int_{\gamma}\left(3 a_{3}+4 a_{2} \xi+2 \xi^{2}\right) d \Psi\right) z^{3}+\ldots\right)+o(\epsilon)
\end{aligned}
$$

on compact subsets.

Hence by Proposition 5.1,

$$
\begin{aligned}
\frac{1}{6} S_{f_{\epsilon}}(0)= & \left(a_{3}+\frac{\epsilon}{2 \pi} \int_{\gamma}\left(3 a_{3}+4 a_{2} \xi+2 \xi^{2}\right) d \Psi\right)- \\
& \left(a_{2}+\frac{\epsilon}{2 \pi} \int_{\gamma} 2\left(a_{2}+\xi\right) d \Psi\right)^{2}+o(\epsilon)
\end{aligned}
$$

Taking the derivative of the new Schwarzian with respect to $\epsilon$, we have

$$
\begin{aligned}
\frac{1}{6} \frac{\partial}{\partial \epsilon} S_{f_{\epsilon}}(0)= & \frac{1}{2 \pi} \int_{\gamma}\left(3 a_{3}+4 a_{2} \xi+2 \xi^{2}\right) d \Psi \\
& -2 a_{2} \frac{1}{2 \pi} \int_{\gamma} 2\left(a_{2}+\xi\right) d \Psi-\frac{2 \epsilon}{2 \pi}\left(\int_{\gamma} 2\left(a_{2}+z\right) d \Psi\right)^{2}+\ldots \\
& +o(1) .
\end{aligned}
$$

When $\epsilon=0$, the derivative becomes

$$
\left.\frac{\partial}{\partial \epsilon} S_{f_{\epsilon}}(0)\right|_{\epsilon=0}=\frac{6}{2 \pi} \int_{\gamma}\left(3 a_{3}-4 a_{2}^{2}+2 \xi^{2}\right) d \Psi .
$$

\section{Reduction to at Most Two Proper Sides}

With these computations, we can now show that any function mapping onto a region with more than two disjoint proper sides cannot be extremal. We do this in two steps, first reducing the extremal domain to one having at most four proper sides, then employing our Step Down Lemma to further reduce the possibilities to at most two proper sides.

LEMMA 6.1. Suppose $f \in H_{n}$ is a hyperbolic convex function onto a region with more than four proper sides, then $f$ cannot be extremal for maximizing the Schwarz norm.

Proof. Suppose $f \in H_{n}$ is extremal, that $\left\|S_{f}\right\|_{\mathbb{D}}=S_{f}(0)$ and that $f$ maps onto a region with more than four proper sides. We will apply our variations to show that $f$ cannot be extremal. 
Recall from equation (5.2) that for either of our variations along a side $\Gamma=f(\gamma)$

$$
\left.\frac{\partial}{\partial \epsilon} S_{f_{\epsilon}}(0)\right|_{\epsilon=0}=\frac{6}{2 \pi} \int_{\gamma}\left(3 a_{3}-4 a_{2}^{2}+2 \xi^{2}\right) d \Psi .
$$

By composing $f_{\epsilon}$ with a rotation (which will not change $S_{f_{\epsilon}}$ ), we may assume

$$
\left.\frac{\partial}{\partial \epsilon} S_{f_{\epsilon}}(0)\right|_{\epsilon=0}=\frac{6}{2 \pi} \int_{\gamma} \operatorname{Re}\left\{3 a_{3}-4 a_{2}^{2}+2 \xi^{2}\right\} d \Psi .
$$

But now notice that $\mathcal{K}(\xi)=3 a_{3}-4 a_{2}^{2}+2 \xi^{2}$ wraps $\{|\xi|=1\}$ twice around the image circle $C$. If $\mathcal{K}(\gamma)$ lies completely in the right half plane, then we push the (proper) side $\Gamma$ out, with $\epsilon>0$ as in Lemma 4.1. Then, since $\operatorname{Re} \mathcal{K}(\gamma)>0$ for $\gamma=f^{-1}(\Gamma)$, we will have that $\int_{\gamma} \operatorname{Re}\left\{3 a_{3}-4 a_{2}^{2}+2 \xi^{2}\right\} d \Psi>0$. Similarly, if $\mathcal{K}(\gamma)$ lies completely in the left half plane, $\Gamma$ can be pushed in so that $\int_{\gamma} \operatorname{Re}\left\{3 a_{3}-4 a_{2}^{2}+\right.$ $\left.2 \xi^{2}\right\} d \Psi<0$.

On the other hand, if $f$ is extremal, then $\left.\frac{\partial}{\partial \epsilon} S_{f_{\epsilon}}(0)\right|_{\epsilon=0}$ must equal 0. Otherwise,

$$
\left\|S_{f}\right\|_{\mathbb{D}}=S_{f}(0)<S_{f_{\epsilon}}(0) \leq\left\|S_{f_{\epsilon}}\right\|_{\mathbb{D}}
$$

for some $\epsilon$ sufficiently near 0 . Thus $\mathcal{K}(\xi)$ must cross the imaginary axis at least once for every proper side.

But as $\xi$ travels along $\partial \mathbb{D}, \mathcal{K}(\xi)$ crosses the imaginary axis at most 4 times. Consequently, the range of our extremal function $f$ can have at most 4 proper sides.

REMARK 6.2. We note that the technique employed thus far is fairly general and can be used to show that an extremal domain for general extremal problems has at most a small number of proper sides. But usually the number of parameters is still too large to completely determine the extremal domain. The following critical Step Down Lemma enables us to further reduce the number of proper sides to the point that the actual maps are computable.

Step Down Lemma. A hyperbolically convex function $f \in H_{n}$ onto a region with more than two proper sides cannot be extremal for maximizing the Schwarz norm.

Proof.

If $f \in H_{n}$ is extremal, then by Lemma 6.1, $f(\mathbb{D})$ can have at most four proper sides. Thus $f \in H_{4} \subsetneq H_{5}$.

If $f(\mathbb{D})$ has exactly four proper sides, then an application of the pigeonhole principle implies that one end point $\mathcal{K}\left(z^{*}\right)$ of one of the $\operatorname{arcs} \mathcal{K}(\gamma)$ must lie in the left half plane. Thus for some $z_{0}$ sufficiently close to $z^{*}$, the image under $\mathcal{K}$ of the subarc $\gamma_{0}$ of $\gamma$ joining $z_{0}$ and $z^{*}$ lies completely in the left half plane. As a result,

$$
\int_{\gamma_{0}} \operatorname{Re}\left\{3 a_{3}-4 a_{2}^{2}+2 \xi^{2}\right\} d \Psi<0
$$

Applying Lemma 4.2 to push the corresponding arc $\Gamma_{0}=f\left(\gamma_{0}\right)$ "in" (varying by $-\epsilon$ ), will then produce both an additional proper side and an increase in the Schwarzian since $-\int_{\gamma} \mathcal{K}(\xi) d \Psi>0$. This varied function $f_{\epsilon}$ will thus lie in $H_{5} \backslash H_{4}$ and have a larger Schwarz norm than any function in $H_{4}$. But this contradicts 
16 Roger W. BARNARD, LEAH COLE, KENT PEARCE, AND G. BROCK WILLIAMS

Lemma 6.1 , since the extremal function in $H_{5}$ can have at most 4 proper sides. As a result, the extremal domain for a map in $H_{5}$ can have at most 3 proper sides.

However, if the extremal domain has exactly 3 proper sides, some endpoint must again lie in the left half-plane. As before, we may add a proper side and increase the Schwarz norm. Thus the extremal domain can have at most two proper sides.

\section{Computing the Schwarz Norms for One- and Two-Sided Functions}

Now we need only compute the Schwarz norm for the remaining possible extremal functions to complete the proof of our theorem. First, however, we need a couple of facts about the behavior of elliptic integrals [1, pp. 53-54].

LEMMA 7.1. If $K$ and $E$ are the complete elliptic integrals of the first and second kind, respectively, then

(i) For each $c \in[1 / 2,2]$, the function $g(x)=\left(\sqrt{1-x^{2}}\right)^{c} K(x)$ is decreasing from $[0,1)$ onto $(0, \pi / 2]$.

(ii) The function

$$
h(t)=\frac{E(t)-\left(1-t^{2}\right) K(t)}{t^{2}}
$$

is increasing from $(0,1)$ onto $(\pi / 4,1)$.

With this lemma, we can prove our main result:

THEOREM 1.1. The maximal value of the Schwarz norm for hyperbolically convex functions is $S_{f_{\alpha}}(0)$, where

$$
f_{\alpha}(z)=\tan \left(\alpha \int_{0}^{z}\left(1-2 \xi^{2} \cos 2 \theta+\xi^{4}\right)^{-1 / 2} d \xi\right), \quad \alpha=\frac{\pi}{2 K(\cos \theta)},
$$

$K$ is the elliptic integral of the first kind, and $\alpha$ is chosen so that $\cos \theta$ is the unique critical point of the function

$$
g(s)=4 s^{2}-2+\frac{\pi^{2}}{2 K^{2}(s)}
$$

on $(0,1)$.

Numerical calculation indicates the extremal value of the Schwarz norm is approximately 2.383635 and is achieved by $S_{f_{\alpha}}(0)$ with $\alpha \approx 0.5343598$.

Proof.

By Lemma 6.1 and the Step Down Lemma, the extremal function in $H_{n}, n>$ 2 , cannot map onto a region with more than 2 proper sides. Thus we need only determine whether the extremal function has 0,1 , or 2 proper sides.

The only hyperbolically convex functions onto a region with no proper sides are disc automorphisms which have Schwarz norm equal to 0.

Any hyperbolically convex function onto a region with exactly one proper side differs from the function $g$ of Example 2.6 by composition with Möbius transfor- 
mations, and hence has the same Schwarz norm, namely 3/2. Note that Mejía and Pommerenke [10] also explicitly computed these maps and their Schwarz norm.

Next notice that for two-sided domains whose proper sides intersect inside the disc, the intersection point can be moved to the origin by a disc automorphism. Moreover, domains bounded by two proper sides which do not intersect can be mapped via a disc automorphism onto an odd symmetric domain, as in figure 2, and domains whose two proper sides intersect on the boundary of the disc can be approximated by domains whose (proper) sides do not intersect. Thus there are two types of regions bounded by exactly two proper sides that we must consider:

(i) Domains in which the two proper sides intersect at the origin

(ii) Odd symmetric domains in which the proper sides do not intersect

For case ((i)), consider the family of maps

$$
g_{\alpha}(z)=\left(\frac{\sqrt{z}-1}{\sqrt{z}+1}\right)^{\alpha}, \quad 0<\alpha<1
$$

from the upper half plane onto a sector of $\mathbb{D}$ with an opening of angle $\alpha \pi$ at the origin. Functions which map onto domains of the first type can be obtained from one of the maps $g_{\alpha}$ by composition with Möbius transformations, thus we may limit our attention to this family.

Computation and simplification reveals

$$
S_{g_{\alpha}}(z)=\frac{3 z^{2}-\left(2+4 \alpha^{2}\right) z+3}{8 z^{2}(z-1)^{2}} .
$$

Because both the Schwarzian and the hyperbolic density $\eta_{\mathbb{H}}(z)=1 /(2 \operatorname{Im} z)$ are invariant under horizontal translation, we need only consider pure imaginary numbers when computing the Schwarz norm. Thus

$$
\begin{aligned}
\left\|S_{g_{\alpha}}\right\|_{\mathbb{H}}= & \sup _{z \in \mathbb{H}} 4(\operatorname{Im} z)^{2}\left|\frac{3 z^{2}-\left(2+4 \alpha^{2}\right) z+3}{8 z^{2}(z-1)^{2}}\right| \\
& =\sup _{y>0} \frac{1}{2} \frac{\left|3 y^{2}+\left(2+4 \alpha^{2}\right) i y-3\right|}{y^{2}+1} .
\end{aligned}
$$

The derivative of the right hand side with respect to $y$ is

$$
\frac{-8\left(\alpha^{2}-1\right)\left(\alpha^{2}+2\right)\left(y^{3}-y\right)}{\sqrt{9 y^{4}+\left(16 \alpha^{4}+16 \alpha^{2}-14\right) y^{2}+9}\left(y^{2}+1\right)^{2}} .
$$

Clearly the denominator is positive for all $y>0,0<\alpha<1$. Moreover, for all $0<\alpha<1$ the numerator is negative for $0<y<1$ and positive for $1<y<\infty$. Consequently, $\eta_{\mathbb{H}}(i y)\left|S_{g_{\alpha}}(i y)\right|$ is decreasing on $0<y<1$ and increasing on $1<y<$ $\infty$. Thus the supremum in the definition of $\left\|S_{g_{\alpha}}\right\|_{\mathbb{H}}$ must occur as $y$ approaches 0 or as $y$ approaches $\infty$. See Figure 6 .

In both directions, however,

$$
\begin{gathered}
\lim _{y \rightarrow 0^{+}} \eta_{\mathbb{H}}(i y)\left|S_{g_{\alpha}}(i y)\right|=\lim y \rightarrow 0^{+} \frac{1}{2} \frac{\left|3 y^{2}+\left(2+4 \alpha^{2}\right) i y-3\right|}{y^{2}+1}=\frac{3}{2} \\
\lim _{y \rightarrow \infty} \eta_{\mathbb{H}}(i y)\left|S_{g_{\alpha}}\right|(i y)=\lim y \rightarrow \infty \frac{1}{2} \frac{\left|3 y^{2}+\left(2+4 \alpha^{2}\right) i y-3\right|}{y^{2}+1}=\frac{3}{2} .
\end{gathered}
$$

Notice that both limits are independent of $\alpha$. Consequently, the Schwarz norm of any hyperbolically convex function onto a domain bounded by exactly two proper sides intersecting inside $\mathbb{D}$ must be $3 / 2$. 


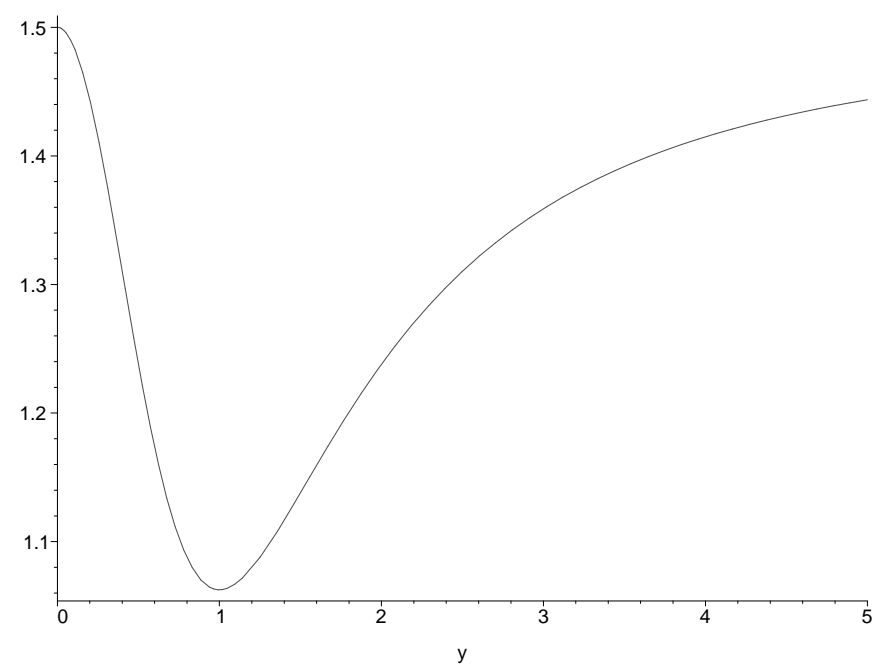

FIGURE 6. The graph of $4 y^{2} \mid S_{g_{\alpha}}($ iy $) \mid$ for $\alpha=0.75$.

The second case consisting of maps onto odd symmetric two-sided domains can be handled using the family of maps $f_{\alpha}$ constructed by Mejía and Pommerenke [10]. Each hyperbolically convex function

$$
f_{\alpha}(z)=\tan \left(\alpha \int_{0}^{z}\left(1-2 \xi^{2} \cos 2 \theta+\xi^{4}\right)^{-1 / 2} d \xi\right)
$$

maps onto a "hyperbolic strip" bounded by two geodesics through

$$
\pm i \tanh \left(\frac{\pi K(\sin \theta)}{4 K(\cos \theta)}\right)
$$

where $\alpha=\frac{\pi}{2 K(\cos \theta)}, 0<\theta<\pi / 2$, and $K$ is the elliptic integral of the first kind. See Figure 2.

For these functions $f_{\alpha}$, our problem is to compute

$$
\left\|S_{f_{\alpha}}\right\|_{\mathbb{D}}=\sup _{z \in \mathbb{D}}\left(1-|z|^{2}\right)^{2}\left|S_{f_{\alpha}}(z)\right| \text {. }
$$

Using an extensive computational argument which considers several cases (various interval ranges for $|z|, \arg z$, and $\alpha$ ) and uses properties of polynomials and $K$, one can show that this problem can be reduced to computing

$$
\sup _{0 \leq x<1}\left(1-x^{2}\right)^{2}\left|S_{f_{\alpha}}(x)\right| \text {. }
$$

Mejía and Pommerenke [10] computed $S_{f_{\alpha}}(x)$ to be

$$
S_{f_{\alpha}}(x)=\frac{2\left(\left(\cos (2 \theta)+\alpha^{2}\right)+\left(\cos ^{2}(2 \theta)-2 \alpha^{2} \cos (2 \theta)-3\right) x^{2}+\left(\cos (2 \theta)+\alpha^{2}\right) x^{4}\right)}{\left(1-2 \cos (2 \theta) x^{2}+x^{4}\right)^{2}}
$$

where $\alpha=\frac{\pi}{2 K(\cos \theta)}$ and conjectured that the maximum value of $\left(1-x^{2}\right)^{2}\left|S_{f_{\alpha}}(x)\right|$ occurs at $x=0$. (Note the typographical error on the second $\alpha$ in the original statement in [10].) 
We claim that maximizing $\left(1-x^{2}\right)^{2}\left|S_{f}(x)\right|$ is equivalent to maximizing $(1-$ $\left.x^{2}\right)^{2} S_{f}(x)$, i.e., we claim that $\max \left(1-x^{2}\right)^{2} S_{f}(x)>-\min \left(1-x^{2}\right)^{2} S_{f}(x)$. We will show that

$$
\min \left(1-x^{2}\right)^{2} S_{f}(x) \geq-2
$$

over $0<x<1$ and $0<\theta<\pi / 2$. Then, as Mejía and Pommerenke [10] noted (and as we will show) that $\max \left(1-x^{2}\right)^{2} S_{f}(x)>2$, the claim will hold.

We simplify our algebraic computations by replacing $x$ with $\sqrt{x}$ in the above expression and obtain

$$
(1-x)^{2} \frac{2\left(\left(\cos (2 \theta)+\alpha^{2}\right)+\left(\cos ^{2}(2 \theta)-2 \alpha^{2} \cos (2 \theta)-3\right) x+\left(\cos (2 \theta)+\alpha^{2}\right) x^{2}\right)}{\left(1-2 \cos (2 \theta) x+x^{2}\right)^{2}}
$$

where $0 \leq x<1$ and $0<\theta<\pi / 2$.

To minimize (7.3), we note that the coefficient of $\alpha^{2}$ is $1-2 \cos (2 \theta) x+x^{2}$ which is nonnegative. Hence, substituting $\alpha=0$ into (7.3), we have that (7.3) is bounded below by

$$
(1-x)^{2} \frac{2\left(\cos (2 \theta)+\left(\cos ^{2}(2 \theta)-3\right) x+\cos (2 \theta) x^{2}\right)}{\left(1-2 \cos (2 \theta) x+x^{2}\right)^{2}}
$$

We will show that (7.4) is bounded below by -2 by showing that

$$
\begin{aligned}
& (1-x)^{2} \frac{2\left(\cos (2 \theta)+\left(\cos ^{2}(2 \theta)-3\right) x+\cos (2 \theta) x^{2}\right)}{\left(1-2 \cos (2 \theta) x+x^{2}\right)^{2}}+2 \\
& =\frac{p(c, x)}{\left(1-2 \cos (2 \theta) x+x^{2}\right)^{2}}>0
\end{aligned}
$$

where

$p(c, x)=\left(2 x+4 x^{2}+2 x^{3}\right) c^{2}+\left(2-12 x+4 x^{2}-12 x^{3}+2 x^{4}\right) c+\left(2-6 x+16 x^{2}-6 x^{3}+2 x^{4}\right)$ and $c=\cos (2 \theta)$ satistifies $-1<c<1$.

It is sufficient to show that $p(c, x)>0$ for $-1<c<1$ and $0<x<1$. We note that $p(c, x)$ is quadratic in $c$ for each fixed $x$ and the coefficent of $c^{2}$ is positive. Let

$$
c(x)=-\frac{2-12 x+4 x^{2}-12 x^{3}+2 x^{4}}{2\left(2 x+4 x^{2}+2 x^{3}\right)},
$$

then $c(x)$ is the location of the vertex of the quadratic $p(c, x)$ for each fixed $x$. We claim that $c(x)$ is an increasing function of $x$ on $(0,1)$. To see this, we note that

$$
c^{\prime}(x)=\frac{n(x)}{2(x+1)\left(1+2 x+x^{2}\right) x^{2}}
$$

where $n(x)=-x^{5}-3 x^{4}+14 x^{3}-14 x^{2}+3 x+1$. Clearly, $n(0)=1$ and $n(1)=0$. A Sturm sequence argument shows that $n(x)$ has exactly one zero on $(0,1]$. Hence, we have $c^{\prime}(x)>0$ on $(0,1)$ and $c(x)$ is increasing on $(0,1)$.

Let $x_{0}$ be the unique solution of $c(x)=-1$ for $0<x<1, x_{0} \approx 0.1197$. For 
$0<x<x_{0}$, the location $c(x)$ of the vertex of $p(c, x)$ satisfies $c(x)<-1$. Hence over $-1<c<1$ and $0<x<1$, we have $\min p(c, x)>p(-1, x)=8 x+16 x^{2}+8 x^{3}>0$. For $x_{0}<x<1$, we have $-1<c(x)<1$ and, hence,

$$
\min p(c, x)=\left.p(c, x)\right|_{c=c(x)}=\frac{q(x)}{2 x\left(1+2 x+x^{2}\right)}
$$

where

$$
q(x)=-x^{8}+16 x^{7}-44 x^{6}+48 x^{5}-3 x x^{4}+48 x^{3}-44 x^{2}+16 x-1 .
$$

Clearly, $q(1)=0$ and $q(1 / 2)=\frac{143}{256}>0$. A Sturm sequence argument shows that on the interval $\left(x_{0}, 1\right]$ that $q$ has exactly one zero. Hence, on $(0,1), q(x)>0$.

Thus, $p(c, x)>0$ for $-1<c<1$ and $0<x<1$ and, consequently, $\min (1-$ $\left.x^{2}\right)^{2} S_{f}(x) \geq-2$.

To maximize (7.3), we substitute $\alpha=\frac{\pi}{2 K(\cos (\theta))}$, take the derivative with respect to $x$, and simplify to obtain

$$
\begin{aligned}
\frac{\left(x^{2}-1\right)(\cos (2 \theta)-1)}{K^{2}(\cos (\theta))\left(x^{2}-2 \cos (2 \theta) x+1\right)^{3}} & \left(K ^ { 2 } ( \operatorname { c o s } \theta ) \left(-10 x^{2} \cos (2 \theta)\right.\right. \\
& -6 x^{2}+12 x \cos (2 \theta)-4 x \cos ^{2}(2 \theta) \\
& \left.+24 x-10 \cos (2 \theta)-6)-\pi^{2}\left(x^{2}-2 \cos (2 \theta)+1\right)\right)
\end{aligned}
$$

Notice that the denominator of this expression is always positive. On the other hand, the numerator changes sign as $x$ varies. However, we will see that for fixed $\theta$, the numerator can change sign only once.

To justify this claim, fix $\theta$ and let

$$
\begin{aligned}
A(x)=K^{2}(\cos \theta)( & -10 x^{2} \cos (2 \theta)-6 x^{2}+12 x \cos (2 \theta)-4 x \cos ^{2}(2 \theta) \\
& +24 x-10 \cos (2 \theta)-6)-\pi^{2}\left(x^{2}-2 \cos (2 \theta)+1\right) .
\end{aligned}
$$

Notice that $\left(x^{2}-1\right)(\cos (2 \theta)-1)>0$ for $0 \leq x<1$ and $0<\theta<\pi / 2$, so we need only show that $A(x)$ changes sign only once.

It follows from known properties [1] of $K$ that $K(\cos (\theta))>\pi / 2$; thus, since the coefficient of $K(\cos (\theta))$ in (7.6) is positive,

$$
\begin{aligned}
A^{\prime}(x) & =K^{2}(\cos (2 \theta))\left(-20 x \cos (2 \theta)-12 x+12 \cos (2 \theta)-4 \cos ^{2}(2 \theta)+24\right)-2 \pi^{2}(x-\cos (2 \theta)) \\
& >\left(\frac{\pi}{2}\right)^{2}\left(-20 x \cos (2 \theta)-12 x+12 \cos (2 \theta)-4 \cos ^{2}(2 \theta)+24\right)-2 \pi^{2}(x-\cos (2 \theta)) \\
& =\left(-10 \pi^{2} \cos ^{2} \theta\right) x+\left(14 \pi^{2} \cos ^{2} \theta-4 \pi^{2} \cos ^{4} \theta\right)
\end{aligned}
$$

The right hand side of the above inequality is linear in $x$ and positive at both $x=0$ and $x=1$. Thus $A^{\prime}(x)>0$ and $A(x)$ is increasing for all $0 \leq x<1$. Consequently, $A(x)$ can change sign at most once.

Notice

$$
A(0)=-10 \cos (2 \theta) K^{2}(\cos (2 \theta))-6 K^{2}(\cos (\theta))-\pi^{2}<0
$$

for all $0<\theta<\pi / 2$.

Thus, as a consequence of the above argument, for each fixed $\theta,\left(1-x^{2}\right)^{2} S_{f_{\alpha}}(x)$ is decreasing at $x=0$ and switches from decreasing to increasing at most once on 


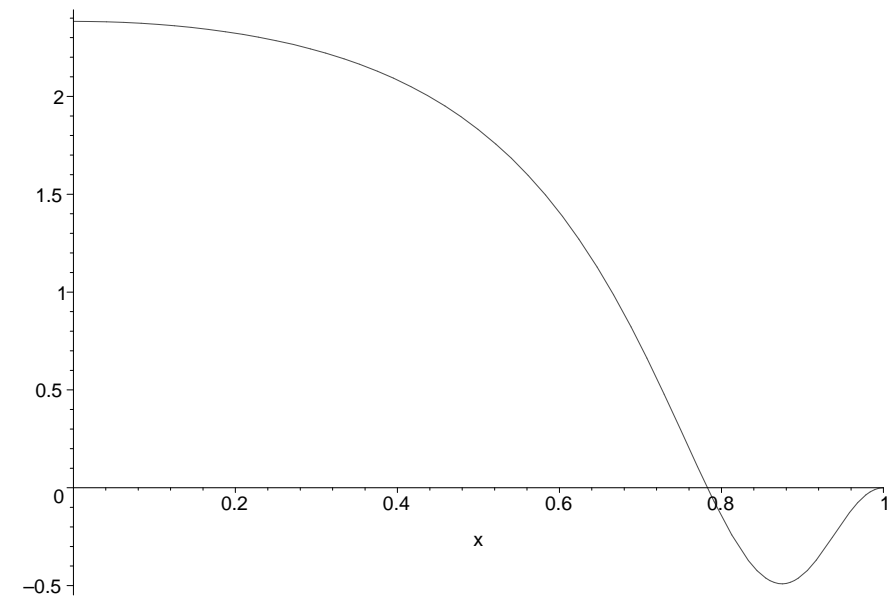

FiguRE 7. The graph of $\left(1-x^{2}\right)^{2} S_{f_{\alpha}}(x)$ for $0 \leq x<1$ and $\alpha=0.5343598$ $(\theta=0.218202)$.

the interval $0 \leq x<1$. (Recall that to establish this fact, we replaced $x$ by $\sqrt{x}$ in our calculations. We now return $x$ to its original meaning.)

Clearly $\left(1-x^{2}\right)^{2} S_{f_{\alpha}}(x)$ equals 0 when $x=1$. Thus even after $\left(1-x^{2}\right)^{2} S_{f_{\alpha}}(x)$ begins increasing, it never becomes larger than 0 .

On the other hand, when $x=0$,

$$
\left.\left(1-x^{2}\right)^{2} S_{f_{\alpha}}(x)\right|_{x=0}=2 \cos (2 \theta)+\frac{\pi^{2}}{2 K^{2}(\cos (\theta))} .
$$

Applying Lemma 7.1 with $c=1 / 2$ we obtain

$$
\begin{aligned}
\left.\left(1-x^{2}\right)^{2} S_{f_{\alpha}}(x)\right|_{x=0} & \geq 2 \cos (2 \theta)+\frac{\pi^{2}}{\frac{\pi^{2}}{2 \sqrt{1-\cos ^{2}(2 \theta)}}} \\
& =2(1+2 \sin (\theta))(1-\sin (\theta))>0
\end{aligned}
$$

for all $0<\theta<\pi / 2$. Thus, $\sup _{0 \leq x<1}\left(1-x^{2}\right)^{2} S_{f_{\alpha}}(x)$ is indeed achieved for $x=0$. See Figure 7.

Now the only question is to determine for which value (or values) of $\theta$

$$
\left\|S_{f_{\alpha}}\right\|_{\mathbb{D}}=S_{f_{\alpha}}(0)=4 \cos ^{2}(\theta)-2+\frac{\pi^{2}}{2 K^{2}(\cos (\theta))}
$$

is maximized. To this end, let $s=\cos \theta$ and let

$$
g(s)=4 s^{2}-2+\frac{\pi^{2}}{2 K^{2}(s)} .
$$

Notice that

$$
\frac{d g}{d \theta}=\frac{d g}{d s} \frac{d s}{d \theta}
$$

and $\frac{d s}{d \theta}=-\sin (\theta)<0$ for $0<\theta<\pi / 2$. Thus since $\cos (\theta)$ is decreasing for 
22 ROGER W. BARNARD, LEAH COLE, KENT PEARCE, AND G. BROCK WILLIAMS

$0<\theta<\pi / 2$, if $\frac{d g}{d s}$ switches signs at most once, then $\left\|S_{f_{\alpha}}\right\|_{\mathbb{D}}$ will have a relative maximum for at most one value of $\theta$.

Computing this derivative and simplifying, we obtain

$$
\frac{d g}{d s}(s)=8 s-\frac{\pi^{2}}{s} \frac{\left.E(s)-\left(1-s^{2}\right) K(s)\right)}{\left(1-s^{2}\right) K^{3}(s)} .
$$

For $0<s<1$, we rewrite $(7.7)$ as

$$
2 s\left(4-\frac{\pi^{2}}{2} \frac{E(s)-\left(1-s^{2}\right) K(s)}{s^{2}} \frac{1}{\left(\left(\sqrt{1-s^{2}}\right)^{2 / 3} K(s)\right)^{3}}\right) .
$$

Applying the the first part of Lemma 7.1 with $c=2 / 3$, we observe that

$$
\frac{1}{\left(\left(\sqrt{1-s^{2}}\right)^{2 / 3} K(s)\right)^{3}}
$$

is increasing for $0<s<1$. Similarly, by the second part of Lemma 7.1,

is increasing for $0<s<1$.

$$
\frac{E(s)-\left(1-s^{2}\right) K(s)}{s^{2}}
$$

Consequently, $\frac{d g}{d s}$ can be written as a product of $2 s$, which is positive, and a factor, which is 4 minus an increasing function. Thus, $\frac{d g}{d s}$ has at most one zero. Moreover, Lemma 7.1 also implies that

$$
\begin{aligned}
\lim _{s \rightarrow 0^{+}} \frac{E(s)-\left(1-s^{2}\right) K(s)}{s^{2}} & =\frac{\pi}{4} \\
\lim _{s \rightarrow 0^{+}}\left(\left(\sqrt{1-s^{2}}\right)^{2 / 3} K(s)\right)^{3} & =\left(\frac{\pi}{2}\right)^{3} .
\end{aligned}
$$

Thus,

$$
\lim _{s \rightarrow 0^{+}} \frac{d g}{d s}=4-\frac{\pi^{2}}{2} \frac{\pi / 4}{(\pi / 2)^{3}}=3 .
$$

On the other hand,

$$
\begin{aligned}
& \lim _{s \rightarrow 1^{-}} \frac{E(s)-\left(1-s^{2}\right) K(s)}{s^{2}}=1 \\
& \lim _{s \rightarrow 1^{-}}\left(\left(\sqrt{1-s^{2}}\right)^{2 / 3} K(s)\right)^{3}=0 .
\end{aligned}
$$

Hence,

$$
\lim _{s \rightarrow 1^{-}} \frac{d g}{d s}=-\infty
$$

Since $\frac{d g}{d s}$ decreases from 3 to minus infinity as $s$ increases from 0 to 1 , we have proven that $\left\|S_{f_{\alpha}}\right\|_{\mathbb{D}}$ does have a unique maxiumum. A Maple computation shows that this maximum is approximately 2.383635 and occurs for $\theta \approx 0.218202$, which corresponds to $\alpha \approx 0.5343598$. See Figure 8 .

Remark 7.2. Mejía and Pommerenke [15] have recently determined the sharp upper bound for $\left|f^{\prime}\left(r e^{i \theta}\right)\right|, 0 \leq r<1$, for hyperbolically convex functions $f$. Applying the techniques of this paper we give another proof and extensively generalize 


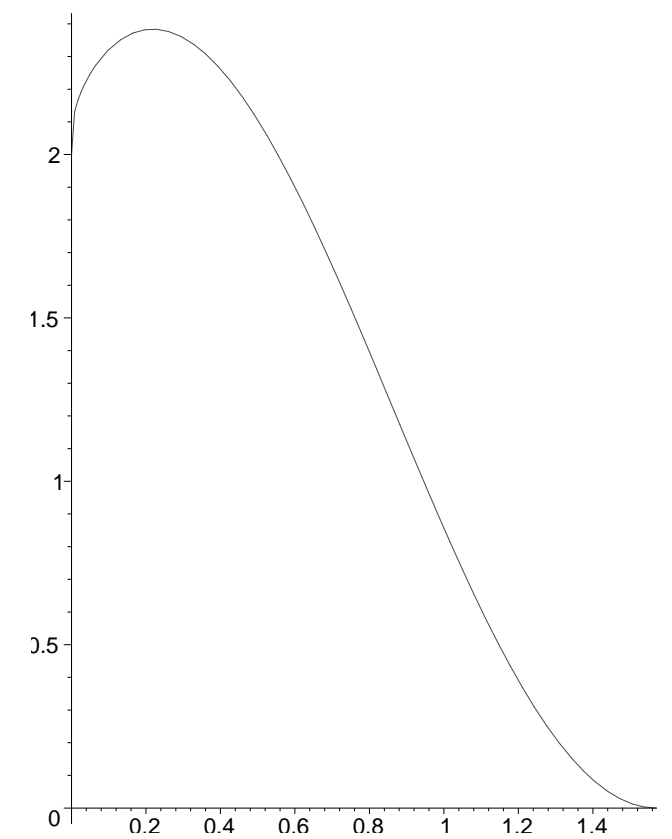

FiguRE 8. The graph of $\left\|S_{f_{\alpha}}\right\| \mathbb{D}$ for $0<\theta<\pi / 2$.

this result [4]. We have also answered in [2] several other related conjectures of Pommerenke [19].

\section{References}

1. G.D. Anderson, M.K. Vamanamurthy, and M.K. Vuorinen, Conformal invariants, inequalities, and quasiconformal mappings, J. Wiley and Sons, 1997.

2. R. W. Barnard, K. Pearce, and G. Brock Williams, Three extremal problems for hyperbolically convex functions, Computational Methods and Function Theory 4 (2004), no. 1, 97-109.

3. Roger Barnard and John L. Lewis, Subordination theorems for some classes of starlike fumctions, Pacific J. Math. 56 (1975), no. 2, 333-366. MR 52 \#721

4. Roger W. Barnard, G. Lenny Ornas, and Kent Pearce, A variational method for hyperbolically convex functions, Complex Variables and Elliptic Equations 51 (2006), no. 4, 313-327.

5. A. Beardon, Geometry of discrete groups, Springer-Verlag, Berlin, 1983.

6. Frederick P. Gardiner and Nikola Lakic, Quasiconformal Teichmüller theory, Mathematical Surveys and Monographs, vol. 76, American Mathematical Society, 2000.

7. O. Lehto, Univalent functions and Teichmüller spaces, Springer-Verlag, Berlin - Heidelberg - New York, 1987.

8. William Ma and David Minda, Hyperbolically convex functions, Ann. Polon. Math. 60 (1994), no. 1, 81-100. MR 95k:30037

9. __ Hyperbolically convex functions. II, Ann. Polon. Math. 71 (1999), no. 3, 273-285. MR 2000j:30020

10. Diego Mejía and Christian Pommerenke, On hyperbolically convex functions, J. Geom. Anal. 10 (2000), no. 2, 365-378.

11. On spherically convex univalent functions, Michigan Math. J. 47 (2000), no. 1, 163-172. MR 2001a:30013

12. S_ Sobre la derivada Schawarziana de aplicaciones conformes hiperbólicamente, Revista Colombiana de Matemáticas 35 (2001), no. 2, 51-60.

13. Hyperbolically convex functions, dimension and capacity, Complex Var. Theory Appl. 47 (2002), no. 9, 803-814. MR 2003f:30017

14. On the derivative of hyperbolically convex functions, Ann. Acad. Sci. Fenn. Math. 27 (2002), no. 1, 47-56. 
15. Diego Mejía, Christian Pommerenke, and Alexander Vasil'ev, Distortion theorems for hyperbolically convex functions, Complex Variables Theory Appl. 44 (2001), no. 2, 117-130. MR 2003e:30025

16. Zeev Nehari, The Schwarzian derivative and schlicht functions, Bull. Amer. Math. Soc. 55 (1949), 545-551. MR 10,696e

17. _ Conformal mapping, Dover, 1952

18. A A property of convex conformal maps, J. Analyse Math. 30 (1976), 390-393. MR 55 \#12901

19. Christian Pommerenke, private communication.

20. Ch. Pommerneke, Boundary behavior of conformal maps, Springer - Verlag, Berlin - Heidelberg - New York, 1992.

21. A. Yu. Solynin, Some extremal problems on the hyperbolic polygons, Complex Variables Theory Appl. 36 (1998), no. 3, 207-231. MR 99j:30030

22. __, Moduli and extremal metric problems, Algebra i Analiz 11 (1999), no. 1, 3-86, translation in St. Petersburg Math. J. 11 (2000), no. 1, 1-65. MR 2001b:30058

Roger W. Barnard, Kent Pearce and G. Brock Williams

Department of Mathematics and Statistics, Texas Tech University, Lubbock, Texas 79409

United States

roger.w.barnard@ttu.edu kent.pearce@ttu.edu brock.williams@ttu.edu
Leah Cole

Department of Mathematics, Wayland Baptist University, Plainview, Texas 79072 United States

colel@wbu.edu 